\title{
Wound Healing Effect of Slightly Acidic Electrolyzed Water on Cutaneous Wounds in Hairless Mice via Immune-Redox Modulation
}

\author{
Hae Sun You, ${ }^{a, b, \#}$ Ailyn Fadriquela, ${ }^{c, \#}$ Ma Easter Joy Sajo, ${ }^{c}$ Johny Bajgai, ${ }^{c}$ Jesmin Ara, ${ }^{c}$ \\ Cheol Su Kim, ${ }^{d}$ Soo-Ki Kim, ${ }^{d}$ Jin Rok Oh, ${ }^{e}$ Kwang Yong Shim, ${ }^{f}$ Hyun Kyo Lim, ${ }^{*, a}$ and \\ Kyu-Jae Lee*,c,g \\ ${ }^{a}$ Department of Anesthesiology and Pain Medicine, Wonju College of Medicine, Yonsei University; Wonju 26426, \\ Korea: ${ }^{b}$ Department of Anesthesiology and Pain Medicine, Korea University Medical Center, Anam Hospital; Seoul \\ 02841, Korea: ${ }^{c}$ Department of Environmental Medical Biology, Wonju College of Medicine, Yonsei University; Wonju \\ 26426, Korea: ${ }^{d}$ Department of Microbiology, Wonju College of Medicine, Yonsei University; Wonju 26426, Korea: \\ ${ }^{e}$ Department of Orthopedics, Wonju College of Medicine, Yonsei University; Wonju 26426, Korea: ${ }^{f}$ Department of \\ Internal Medicine, Wonju College of Medicine, Yonsei University; Wonju 26426, Korea: and ${ }^{g}$ Institute for Poverty \\ Alleviation and International Development, Yonsei University; Wonju Campus, Wonju 26493, Korea.
}

Received March 14, 2017; accepted June 12, 2017

\begin{abstract}
Acidic electrolyzed water is an innovative sanitizer having a wide-spectrum of applications in food industry, and healthcare industry but little is known on its effect and mechanism in wound healing. The study was conducted to identify the effect and mechanism of slightly acidic electrolyzed water (SAEW) on cutaneous wounds in hairless mice. SAEW (pH: 5-6.5, oxidation reduction potential: $800 \mathrm{mV}$, chlorine concentration: $25 \mathrm{ppm}$ ) was prepared through electrolysis of water and was applied to the wounds of hairless mice three times a day for seven days. Wound size, immune response and oxidative stress were explored and compared to conventional agents such as Betadine and alcohol. We found that SAEW-treated group showed the highest wound reduction percentage $(p<0.01)$. Antioxidant activities such as glutathione peroxidase, catalase and myeloperoxidase activities of SAEW group surpassed the total reactive oxygen species in skin. Nuclear factor erythroid-2-related-factor-2 and aryl hydrocarbon receptor were upregulated in SAEW group. Further, SAEW recruited the production of intracellular calcium and promoted its utilization for faster healing. In line, SAEW treatment decreased pro-inflammatory cytokines [interleukin (IL)-1/, IL-6, keratinocyte chemoattractant, and tumor necrosis factor- $\alpha$ ] in serum. Other hallmarks of wound healing, matrixmetalloproteinases (MMP)1 and MMP9 were also upregulated. Collectively, our study indicates that SAEW is effective in wound healing of hairless mice via immune-redox modulation, and heals better/faster than conventional agents.
\end{abstract}

Key words slightly acidic electrolyzed water; wound healing; oxidative stress; immune response

Skin injuries, such as cutaneous wounds, need to undergo a complex mechanism to repair the damage. Wound healing is comprised of four sequential but overlapping stages; homeostasis, inflammation, proliferation, and remodeling, strongly regulated with the goal of restoring the integrity of the skin. ${ }^{1)}$ Several studies reveal that different factors can influence wound healing such as immune response and inflammation, redox homeostasis, and also $\mathrm{pH}^{2-5)}$ In relation to this, there is a rise in the use of topical applications or dressings to control and alter skin $\mathrm{pH}$ which can make wounds heal faster. The use of alcohol and povidone-iodine (Betadine) are some of the proven and tested agents in wound healing. Different phytoextracts are being used and currently being evaluated for their wound healing efficacy. ${ }^{6}$ However, some of those treatments can be expensive and can possess side effects.

Acidic electrolyzed water (AEW) is produced by electrolysis of water. It is produced by a machine where water and low concentration of solution (i.e., $\mathrm{HCl}$ or $\mathrm{NaCl}$ ) pass through and generates water containing oxygen gas, and available chlorine concentration such as $\mathrm{Cl}_{2}, \mathrm{HClO}, \mathrm{ClO}^{-}$as components. ${ }^{7,8)}$ This generally corresponds to acidic water with a general characteristic of having low $\mathrm{pH}(2.2-6.5)$, high oxidation-reduction potential $(800-1100 \mathrm{mV})$, high available chlorine concentration

\footnotetext{
\# These authors contributed equally to this work.
}

(10-60 ppm) and high content of dissolved oxygen. ${ }^{8)}$ Spectroscopic analysis revealed that acidic water has microbial activity and is maximum between $\mathrm{pH} 4$ and 5, wherein hypochlorous acid also becomes maximum in killing Escherichia coli and Bacillus subtilis. ${ }^{7)}$ Furthermore, acidic water has been known to inactivate microbes, fungus, viruses, and toxins in vitro, and it is also used for disinfecting food equipments, vegetables, fruits, poultry and meat. ${ }^{8)}$ It is also studied for its efficacy on hand-washing, hospital bactericidal effect, and even cleaning medical apparatus such as endoscope. ${ }^{9-11)}$ Single study has also been done wherein electrolyzed water functions as a bactericide in burn injury with Pseudomonas aeruginosa infection in a rat burn-wound model. ${ }^{12)}$

With this in mind, the development and current applications of acidic water in the medical field could be further explored. Current in vitro studies hinted that functional acidic water might be correlated to epithelial signalling pathway regarding $\mathrm{hBD} 2$ and nuclear factor-kappa $\mathrm{B}(\mathrm{NF}-\kappa \mathrm{B})$ regulation on human oral squamous cell carcinoma. ${ }^{13,14)}$ This signal path might be plausible to elucidate the molecular mechanism of acidic water on wound healing. Up to date, three studies have been involved in using electrolyzed water in wound healing. An in vivo study mentioned above was performed to test the effect of acidic water in burn-wound with bacterial infection, and results showed that the mortality rate was lowest in the

* To whom correspondence should be addressed. e-mail: hyunkyolim@yonsei.ac.kr; medbio9@gmail.com 
acidic water group. ${ }^{12}$ Another study testing different waters concluded that the wound healing effect may be due to the free radicals, and the other in vivo study proposed that acidic water enhances epithelialization, collagen deposition and increase of inflammatory cells. ${ }^{15,16)}$ Despite these studies in relation to wound healing, the exact mechanism of the healing effect is still unknown.

This study was performed to investigate the healing effect of slightly acidic electrolyzed water (SAEW) on the cutaneous wound in hairless mice, and if so, how. Different mechanisms involved in wound healing process such as oxidative stress, immune response and other plausible pathways were explored. Further, we compared the efficacy of SAEW with other conventional disinfectants such as Betadine and alcohol.

\section{MATERIALS AND METHODS}

Preparation of SAEW SAEW was generated from an electrolyzing apparatus (HOCLER Cosmic Round Korea Co., Ltd., Seongnam, Korea). SAEW was prepared by electrolysis of tap water mixed with $4.5 \% \mathrm{HCl}$ solution in an electrolytic cell without diaphragm. Tap water had a $\mathrm{pH}$ of 7.0, oxidation reduction potential (ORP) of approximately $400 \mathrm{mV}$ and chlorine concentration of $3.0 \pm 0.5 \mathrm{ppm}$. SAEW produced had properties of $\mathrm{pH} 5.5 \pm 0.5$, ORP of approximately $800 \mathrm{mV}$ and available chlorine concentration of $25.0 \pm 0.2 \mathrm{ppm}$ (mainly hypochlorous acid). The main component of this water was $98-100 \%$ of $\mathrm{HOCl}$ and $0-2 \%$ of $\mathrm{NaOCl}$. Saline solution (Life Science Co., Ltd., Dangjin, Korea), Betadine (10\% povidoneiodine) (Green Pharmacy Inc., Jincheon, Korea), and 70\% alcohol (SK Chemical, Ulsan, Korea) were prepared as control groups.

Animal Groupings Ten-week-old female hairless mice $(n=50)$ weighing $18 \pm 2 \mathrm{~g}$ were purchased (Orient Bio Inc., Seongnam, South Korea) and kept at $22 \pm 2^{\circ} \mathrm{C}$ and $40-60 \%$ humidity under a cycle of $12: 12 \mathrm{~h}$ light and dark. The mice were put in plastic cages (W $172 \times \mathrm{D} 240 \times \mathrm{H} 129 \mathrm{~mm}$ ) with five mice in one cage, were acclimatized for one week, and were assigned randomly to five groups: No wound induction: Normal control (NC) group $(n=10)$, Wound-induced groups; saline-treated group $(n=10)$, Betadine-treated group $(n=10)$, alcohol-treated group $(n=10)$ and SAEW-treated group $(n=10)$. The animal use and protocol was approved by the Institutional Animal Care and Use Committee (IACUC), Yonsei University Wonju Campus (YWC-150827-2).

Wound Induction and Treatment Mice were anesthetized using Isoflurane (Piramal Critical Lab., Inc., PA, U.S.A.) and wounds were induced using $5 \mathrm{~mm}$ biopsy punch (IntegraMiltex, PA, U.S.A.), creating a total of six wounds equally distributed on the dorsal part of the mice. Treatment was done by spraying $2 \mathrm{~mL}$ of the treatment groups on the wounds 3 times a day for $7 \mathrm{~d}$.

Wound Size Measurement and Gross Examination The length and width of two wounds at the middle were measured daily using a vernier scale (Mitutoyo Corp., Japan). Wound area reduction was calculated using the following formula: Wound contraction $(\%)=100 \times[$ (zero day wound size-specific day wound size)/zero day wound size]. ${ }^{17)}$ Gross morphology was observed by taking a photo of the wounds using a digital camera at a constant focusing distance every day for $7 \mathrm{~d}$ to check and compare the wound state of each mouse.
Sample Preparation Serum was prepared after the blood collection and was put into BD Microtainer tube (Becton, Dickinson and Company, Franklin Lakes, NJ, U.S.A.), was centrifuged at $14000 \mathrm{rpm}$ for $5 \mathrm{~min}$ at $4^{\circ} \mathrm{C}$ to get the serum and was kept in at $-80^{\circ} \mathrm{C}$ until further use.

Skin tissues $(1 \times 1 \mathrm{~cm})$ were cut from the wound area of the mice. The skin tissue was placed in ice-cold RIPA buffer (Pierce Biotechnology Inc., IL, U.S.A.) with protease inhibitor cocktail (Sigma Chemical Co., St Louis, U.S.A.) and was homogenized at $25 \mathrm{rpm}$ for $15 \mathrm{~min}$. Thereafter, the crude skin lysate was centrifuged at $14000 \mathrm{rpm}$ for $10 \mathrm{~min}$ at $4^{\circ} \mathrm{C}$ and the supernatant was collected. Skin lysate was checked for protein concentration by Pierce BCA Assay Kit (Thermo Scientific, Rockford, IL, U.S.A.).

White Blood Cells (WBC) and Their Differential Counts On the 7th day of treatment, blood was collected from the retro-orbital plexus and was put in tubes coated with ethylenediaminetetraacetic acid (EDTA). Total WBC and its differential counts (neutrophils, lymphocytes and monocytes) were measured using an automatic blood analyzer (HEMAVET HV950 FS, Drew Scientific Inc., Dallas, TX, U.S.A.).

Reactive Oxygen Species (ROS) and Antioxidant Enzymes Assays Total ROS was measured using 2',7'-dichlorodihydrofluorescin diacetate (DCFH-DA) (Abcam, Cambridge, MA, U.S.A.) by following manufacturer's manual. In brief, $50 \mu \mathrm{L}$ of samples were put in the 96-well plate. One hundred microliters of $10 \mu \mathrm{M}$ DCFH-DA was added and the plate was incubated for $30 \mathrm{~min}$ in the dark. Fluorescence at $488 \mathrm{~nm}$ excitation $/ 525 \mathrm{~nm}$ emission was analyzed using DTX-880 multimode microplate reader (Beckman Counter Inc., Fullerton, CA, U.S.A.).

The activity of superoxide dismutase (SOD), glutathione peroxidase (GPx), catalase (CAT), and myeloperoxidase (MPO) in skin lysate were measured using Biovision kit (Milpitas, CA, U.S.A.) and following each of the manufacturer's instruction. In brief, the normalized protein concentrations together with each reaction mix and solutions were used to measure the activities of different antioxidant enzymes and was analyzed at the following observance: SOD (450 nm), GPx (340 nm), CAT (510 nm), and MPO (412 nm).

Nitric Oxide (NO) Production NO production was measured using Griess reagent (Promega Corp., Madison, WI, U.S.A.) and the assay was done according to manufacturer's instruction. Briefly, $50 \mu \mathrm{L}$ of the reagent was added to the same volume serum in a $96-$ well plate and incubated at room temperature for $15 \mathrm{~min}$. The absorbance was analyzed at $540 \mathrm{~nm}$ using a DTX-880 multimode microplate reader (Beckman Counter Inc., Fullerton, CA, U.S.A.).

Cytokine Analysis Inflammatory cytokines such as interleukin (IL)-1 $\beta$, IL-6, keratinocyte chemoattractant (KC), and tumor necrosis factor (TNF)- $\alpha$ in serum were analyzed using a Bead Array Suspension Multiplex Kit (Bio-Rad, San Diego, CA, U.S.A.) according to the manufacturer's instructions. The plate was run on a Luminex 200 Bio-Plex Instrument (BioRad, Hercules, CA, U.S.A.). Raw data were analyzed by the software using 5-parameter logistic method.

Total Calcium Assay The total calcium concentration was measured using Cayman Calcium Assay Kit (Ann Arbor, MI, U.S.A.), following manufacturer's instructions. Calcium standard in two-fold dilution was prepared and added to the microplate well. Samples were added into the wells followed by the working detector reagent. The plate was incubated for 

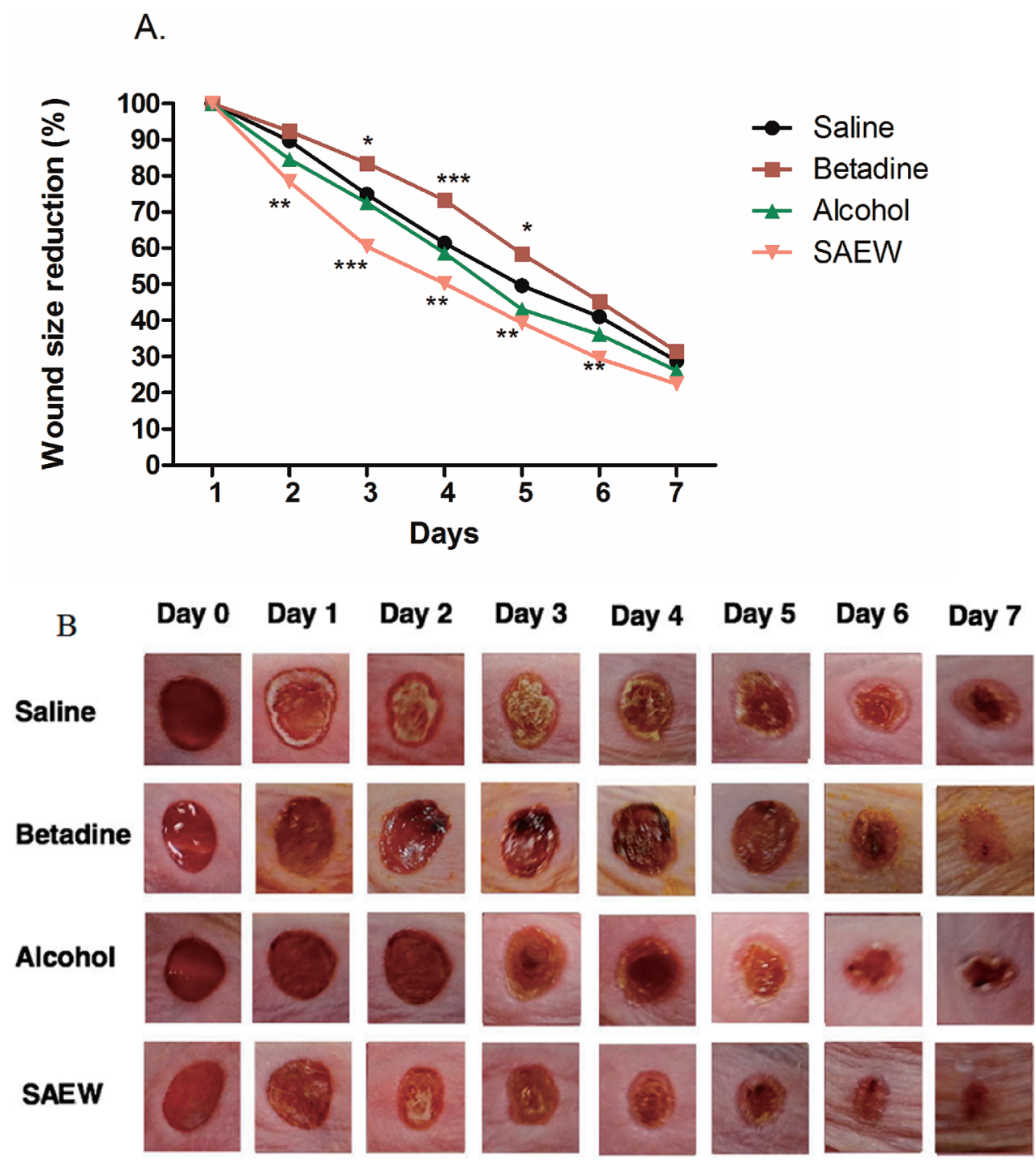

Fig. 1. Wound Area Reduction (A) and Wound Morphology (B) of Different Treatment Groups from Days 1 to 7

All values are presented as mean \pm S.D., $n=10 .{ }^{*} p<0.05, * * p<0.01,{ }^{* * *} p<0.001$ compared with saline group.

$5 \mathrm{~min}$ and was analyzed at $590 \mathrm{~nm}$ (Biotech Instrument Inc., VT, U.S.A.).

Western Blotting The prepared skin lysate with the normalized protein concentration was equally loaded and separated by electrophoresis on sodium dodecyl sulfate-polyacrylamide gels, was transferred to nitrocellulose membranes, and was blocked in 5\% skim milk. Then, the membranes were incubated with primary antibodies overnight at $4^{\circ} \mathrm{C}$, and were incubated with horseradish peroxidase (HRP)-conjugated secondary antibodies for $1 \mathrm{~h}$ at room temperature. Chemiluminescent detection was done using the Chemiluminescence Western blot Detection System (BioSpectrum ${ }^{\circledR} 600$ Imaging System,Upland, CA, U.S.A.). Primary antibodies used for housekeeping: B-actin; for oxidative stress related mechanism: nuclear factor (erythroid-derived 2)-like 2 (Nrf2) (Cell Signaling, Danvers, MA, U.S.A.) and aryl hydrocarbon receptor (AHR) (Abcam); and for remodeling stage mediation: Matrix metalloproteinases (MMP)1 and MMP9 (Abcam). Immunoblotting was done three times in each target protein to confirm results and check statistical significance.

Statistical Analysis The mean values among the groups were analyzed by GraphPad Prism version 5.0 software pack- ages (GraphPad, La Jolla, CA, U.S.A.) and were compared using one-way ANOVA followed by subsequent multiple comparison test (Tukey). A probability value of $p<0.05$ was considered to be significant.

\section{RESULTS}

Wound Size and Gross Morphology To check the wound healing efficacy of SAEW, the wound size reduction was calculated and gross examination was observed for $7 \mathrm{~d}$. The result shows that Betadine-treated group showed an increased wound area as compared to saline group. While saline and alcohol-treated groups had a decreased wound area, SAEWtreated group showed the highest percentage of wound reduction from days 2 to 7 (Fig. 1A). The wound size reduction was significantly reduced on the third day $(p<0.001)$. By the end of day 7, the wound size of SAEW-treated group was reduced to $22.4 \%$, as compared to $26.1 \%$ of alcohol, $31.4 \%$ of Betadine and $28.8 \%$ of saline-treated groups. The digital photograph of a wound representative (Fig. 1B) was consistent with the result as shown in the morphology of the wounds among treatment groups. 
A

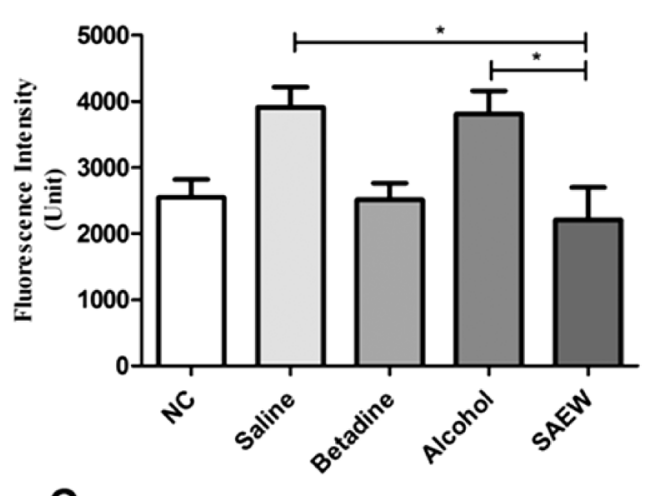

C

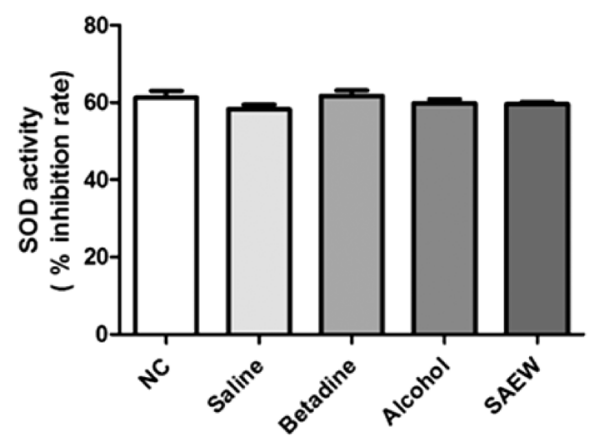

E



B

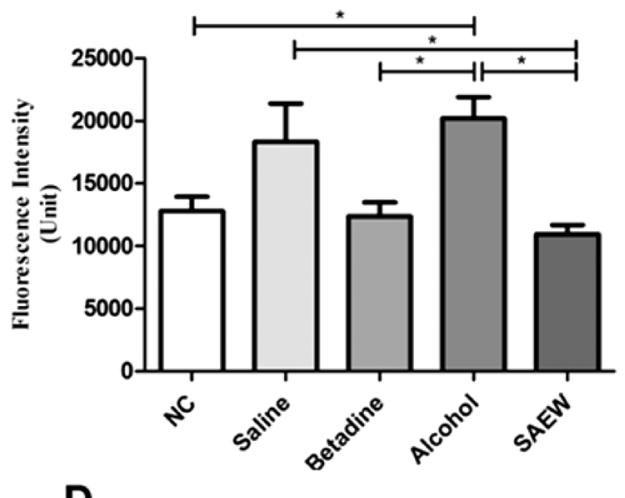

D

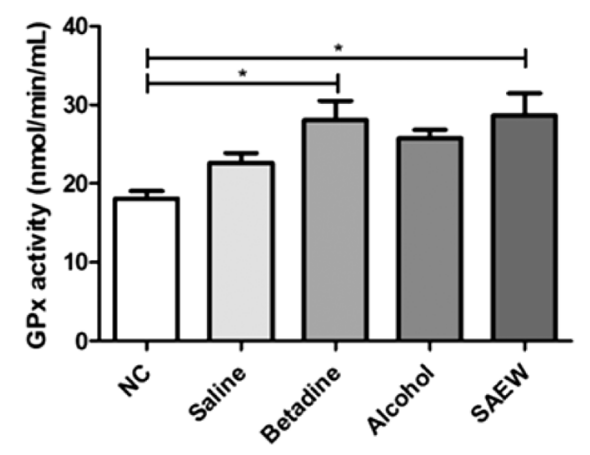

$\mathbf{F}$

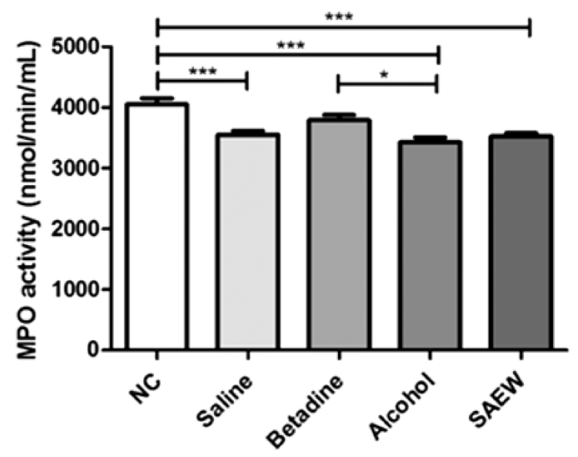

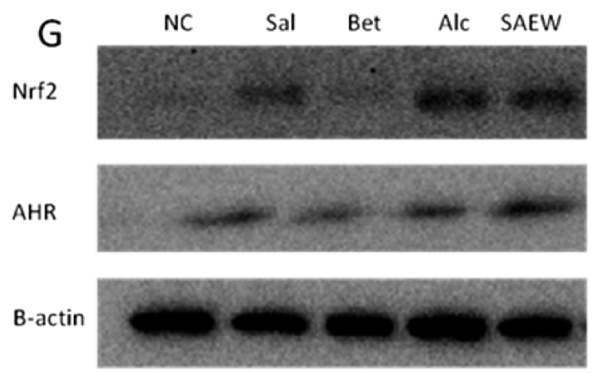
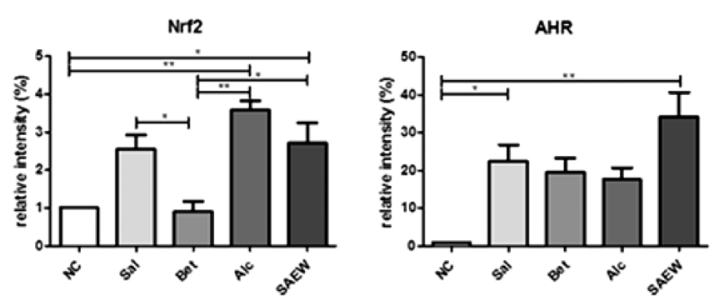

Fig. 2. ROS Levels in Serum (A) and Skin Lysate (B), Skin Lysate's SOD (C), GpX (D), CAT (E) and MPO (F) Levels among Treatment Groups

All values are presented as mean \pm S.D., $n=10 .{ }^{*} p<0.05,{ }^{* *} p<0.01$ and ${ }^{* * *} p<0.001$ indicate significant differences when tested with ANOVA. Tukey's test was used for post-hoc tests. (G) Expression of Nrf2 and AHR on skin lysate of different treatment groups. NC: normal control, Sal: saline, Bet: Betadine, Alc: alcohol, SAEW: slightly acidic electrolyzed water.

Oxidative Stress and Antioxidant Activities ROS and antioxidant enzymes play pivotal roles in wound healing, specifically in defence from pathogens. To identify whether these molecules would affect would healing of SAEW, the levels of ROS and antioxidant enzymes were quantitated. We found that there was a significantly lower ROS level in SAEW as compared to the alcohol-treated group $(p<0.05)$ and saline-treated group $(p<0.05)$ (Fig. 2A). A similar trend was 
A

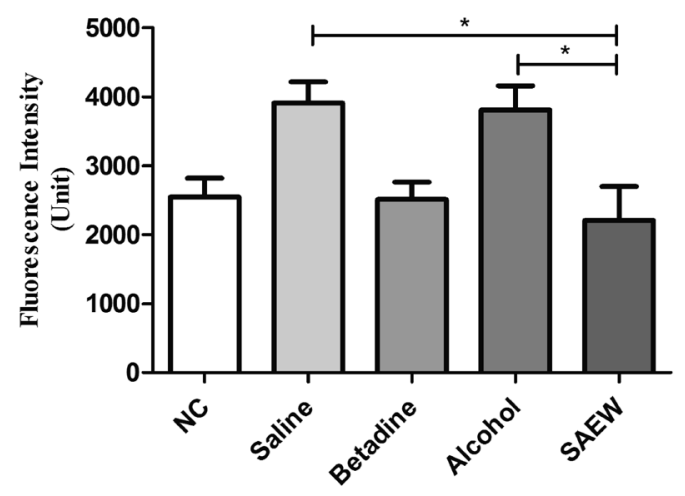

B

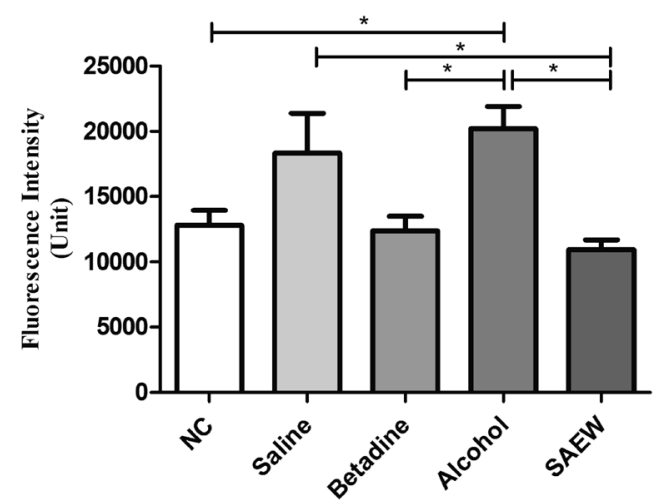

Fig. 3. NO Levels in Serum (A) and Lysate (B)

$* p<0.05$ indicates significant differences when tested with ANOVA. Tukey's test was used for post-hoc tests. NC: normal control, SAEW: slightly acidic electrolyzed water.

Table 1. Total White Blood Cells (WBC) and WBC Differential Counts

\begin{tabular}{llllll}
\hline \hline WBC count $(\mathrm{K} / \mu \mathrm{L})$ & NC & Saline & Betadine & Alcohol \\
\hline Total WBC & $5.14 \pm 0.95$ & $4.04 \pm 0.40^{*}$ & $3.88 \pm 0.58^{* *}$ & $4.02 \pm 0.63^{*}$ \\
Neutrophil & $1.50 \pm 0.28$ & $1.66 \pm 0.13$ & $1.54 \pm 0.18$ & $1.77 \pm 0.39$ \\
Lymphocyte & $3.24 \pm 0.80$ & $2.12 \pm 0.34 * * 1.07 \pm 0.74 *$ & $1.90 \pm 0.42 * * *$ & $2.04 \pm 0.39 * *$ \\
Monocyte & $0.41 \pm 0.09$ & $0.26 \pm 0.07 * * *$ & $0.30 \pm 0.07 *$ & $2.09 \pm 0.59 * *$ & $0.19 \pm 0.05 * * *$ \\
\hline
\end{tabular}

Data were expressed as mean \pm S.D., $n=10$. NC: normal control; SAEW: slightly acidic electrolyzed water. $* p<0.05, * * p<0.01$ and $* * * p<0.001$ compared with NC group, indicates significant differences with ANOVA. Tukey's test was used for post hoc tests.

observed in ROS level of skin lysate comparing SAEW with alcohol-treated $(p<0.05)$ and saline-treated group $(p<0.05)$ (Fig. 2B).

To know the antioxidant activity upon treatment, SOD, GPx, CAT and MPO assays were performed. SOD showed no significant changes among all the treatment groups (Fig. 2C). However, GPx increased among all groups but were significantly higher in Betadine and SAEW-treated groups as compared to NC group $(p<0.05)$ (Fig. 2D). It is also observed that there was no significant change observed between the positive control groups and experimental groups as compared to the saline-treated group. On the other hand, there was a significant reduction of CAT in Betadine-treated $(p<0.05)$ and SAEW-treated $(p<0.001)$ versus NC group and SAEW-treated group also had significantly lower CAT activity as compared to saline-treated group $(p<0.001)$ (Fig. 2E). MPO also showed significant reduction of expression upon treatment of saline, alcohol and SAEW $(p<0.001)$ but other groups observed no significant difference when compared with saline-treated group (Fig. 2F). To further elucidate the oxidative stress pathway, Nrf2, a main regulator of antioxidant enzyme production, and AHR, a protein with adaptive response in oxidative stress, were also checked. Nrf2 level in wounds treated with SAEW was upregulated as compared to NC group $(p<0.05)$ but not statistically different compared to saline-treated group. Similarly, AHR was also more expressed in SAEW treated group as compared to NC group $(p<0.01)$, and an increasing trend was also observed though not statistically significant between SAEW and saline-treated groups (Fig. 2G).

NO Production NO also plays a role in both oxidative stress and inflammation related pathway. NO assay was done to check the effect of the treatment groups on its activity. The results show that NO levels in serum were high in saline and Betadine treated groups, where in alcohol treated group was significantly lower than saline-treated group $(p<0.001)$ but SAEW-treated groups were not statistically different (Fig. 3A). In addition, NO in skin lysate showed a trend of a slight increase in SAEW-treated group as compared to saline-treated group but generally did not show any significant difference among all treatment groups (Fig. 3B).

Immune Modulation To identify how SAEW mediates the immune response which can contribute in its rapid healing process, WBC and its differential counts were analyzed. Table 1 shows that there was a significant reduction of the total WBC in all treatment groups (saline, alcohol and SAEWtreated groups $(p<0.05)$ and Betadine-treated group $(p<0.01)$. Also, there was no significant difference in neutrophil counts among all groups. Lymphocytes of saline, alcohol and SAEWtreated groups were similarly reduced $(p<0.01)$ and Betadinetreated group was greatly reduced $(p<0.001)$. In addition, upon treatment of saline, alcohol and SAEW, monocytes were also significantly reduced $(p<0.001)$ and Betadine-treated group had also decreased monocyte counts $(p<0.05)$. In addition, there was no observed statistical difference between saline-treated group and positive control groups and experimental group.

In line, the production of cytokines is also important in immune response and wound healing. To check their activity and function upon treatment, a multiplex assay was done to test different inflammatory cytokines in serum and was compared among the groups. IL-1 $\beta$ showed a significant reduction in alcohol and SAEW-treated groups $(p<0.001)$ as compared with $\mathrm{NC}$ group and also the saline-treated group (Fig. 4A). A similar trend was seen in the expression of IL-6, wherein alcohol 
A



C

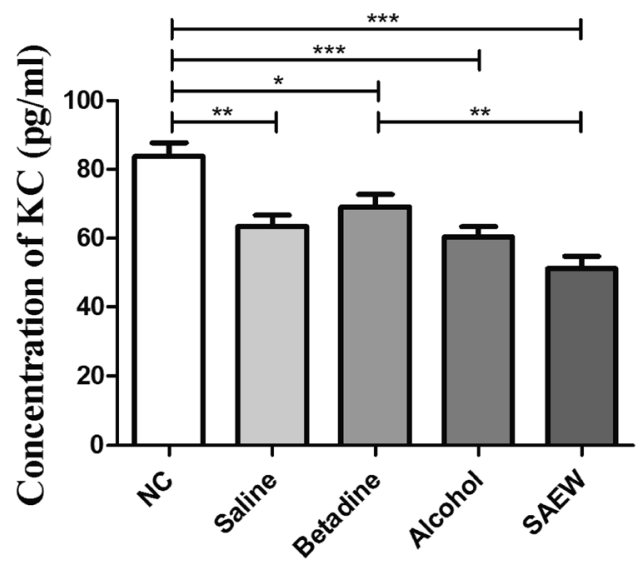

B

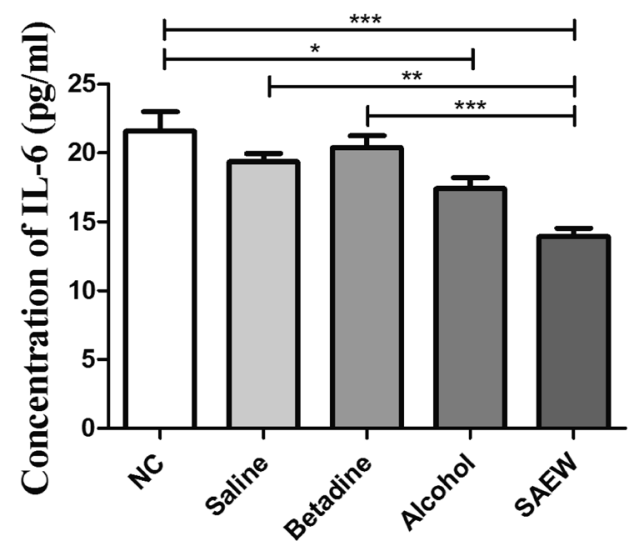

D

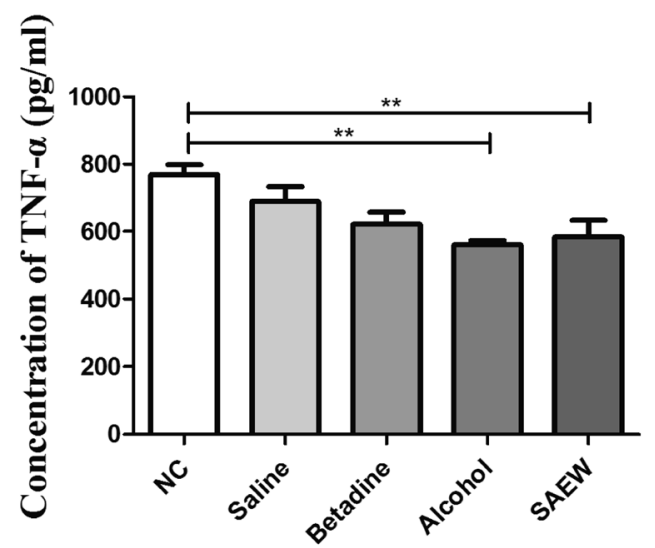

Fig. 4. Effect of the Treatment Groups on IL-1 $\beta$ (A), IL-6 (B), KC (C) and TNF- $\alpha$ (D)

All values are presented as mean \pm S.D., $n=10 . * p<0.05, * * p<0.01$, and $* * * p<0.001$ indicate significant differences when tested with ANOVA. Tukey's test was used for post-hoc tests. NC: normal control, SAEW: slightly acidic electrolyzed water.

A

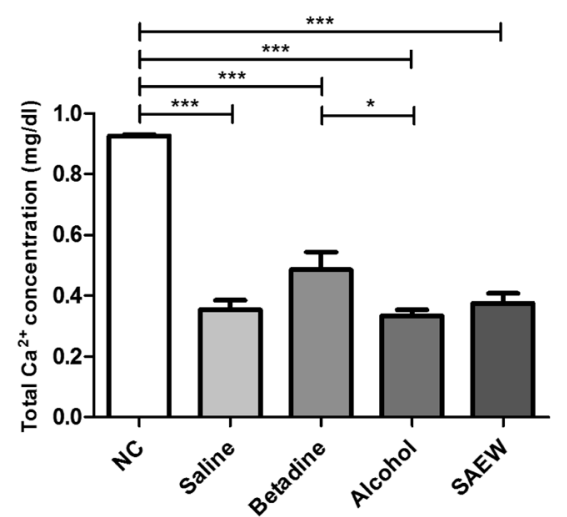

B

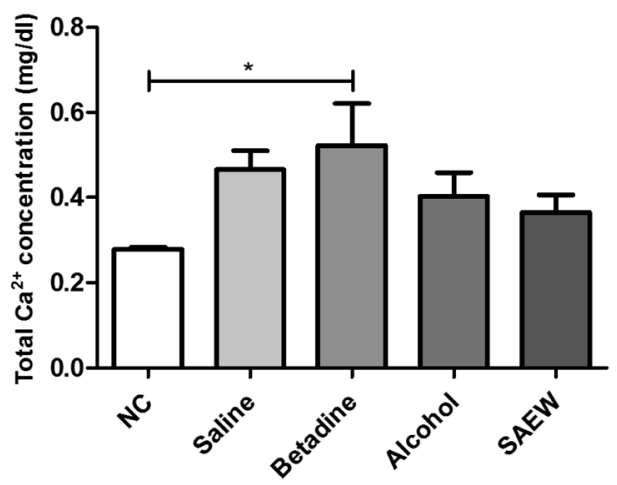

Fig. 5. Intracellular Calcium Concentration of Serum (A) and Skin Lysate (B) among All the Treatment Groups

All values are presented as mean \pm S.D., $n=10$. $* p<0.05$ and $* * * p<0.001$ indicate significant difference when tested with ANOVA. Tukey's test was used for post-hoc tests. NC: normal control, SAEW: slightly acidic electrolyzed water.

and SAEW-treated groups were also significantly reduced $(p<0.05$ and $p<0.001)$ versus NC group and IL- 6 expression was also significantly lower in SAEW-treated group than that of saline-treated group $(p<0.01)$ (Fig. 4B). KC levels of all treatment groups were also reduced significantly especially upon alcohol and SAEW treatment $(p<0.001)$ as compared 

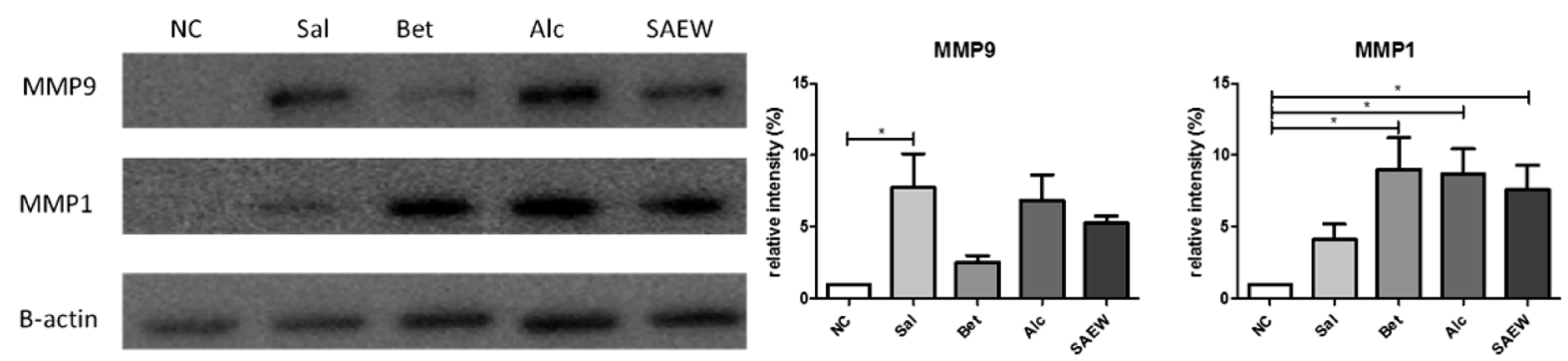

Fig. 6. Expressions of MMP1 and MMP9 in All Treatment Groups

NC: normal control, Sal: saline, Bet: Betadine, Alc: alcohol, SAEW: slightly acidic electrolyzed water. $* p<0.05$ indicates significant differences when tested with ANOVA.

to NC group but no statistical difference was observed when compared to saline-treated group (Fig. 4C). Consistently, TNF- $\alpha$ (Fig. 4D) showed significant reduction in alcohol and SAEW-treated groups $(p<0.01)$ versus NC group but no significant difference observed versus saline-treated group.

Intracellular Calcium Activity Calcium is a central regulator of skin homeostasis and is also involved in wound healing through ROS production. To find out how SAEW could have an effect on its role in wound repair, intracellular calcium were assayed in both serum and skin lysate. Intracellular calcium concentration in serum showed significant reduction among all treated groups $(p<0.001)$ versus $\mathrm{NC}$ group, and alcohol treated groups showed significant reduction as compared to Betadine treated group $(p<0.05)$ while there was no observed significant difference between saline-treated and SAEW-treated groups (Fig. 5A). Calcium concentration on skin lysate, however, showed an increase among all groups but with no observed stastistical significance. However, it is also noted that SAEW had the lowest calcium concentration among all treatment groups.

MMP Production MMPs, important markers in the remodeling stage of wound healing, were also checked. Western blot results showed that MMP1 and MMP9 were upregulated in all groups (Fig. 6) as compared to unwounded mice. In comparison to saline-treated group, MMP1 showed more protein expression while MMP9 showed similar or less expression but not statistically significant.

\section{DISCUSSION}

Our study indicates that SAEW is effective in wound healing of hairless mice via immune-redox modulation, and heals better/faster than conventional agents such as alcohol and Betadine. This was evidenced by the wound size reduction and gross morphology. Wound healing stages such as hemostasis, inflammation, proliferation and remodeling are vital processes to understand the mechanism of restoring the skin. These processes are influenced by different factors, one of which is the difference in $\mathrm{pH}$ by affecting angiogenesis, collagen formation, macrophage and fibroblast activity, keratinocyte proliferation and effects on enzyme activities. ${ }^{18)}$ Taken together, SAEW appears to have a favourable condition that affects effective healing of cutaneous wounds via immuneredox modulation.

One factor essential to wound healing is redox homeostasis. It might be achieved by controlling the balance between ROS production and antioxidant scavenging. The balance of ROS levels is required to escape damaging effects as well as to execute its beneficial functions. ${ }^{4)}$ ROS' role in the inflammatory phase is well known wherein inflammatory cells migrate to the wound and secretes ROS to help in attacking pathogens, and moderate amount of ROS could facilitate angiogenesis and re-epithelialization. ${ }^{3)}$ Based on these, we can infer the potential intervention of SAEW in ROS production from SAEW-treated group having the lowest ROS concentration in both serum and lysate. It shows that upon treatment of SAEW, ROS in the whole body and the wound area are in a controlled amount. Therefore, SAEW produced a desired amount of ROS as compared to alcohol and saline treated groups for its functional roles. These roles can be attributed to the involvement of a low level of ROS in migration and proliferation ${ }^{19)}$ and angiogenesis. $^{20)}$

In addition, antioxidant enzymes play a role in detoxification of ROS in the skin to avoid oxidative stress and to maximize the healing effect. ${ }^{4}$ In this study, SOD did not have any significant change which is evidence that the step toward the dismutation of superoxide ion into $\mathrm{H}_{2} \mathrm{O}_{2}$ was already done. This result confirms that the wounds were past initial inflammatory phase of wound healing. CAT and GPx, on the other hand, showed some significant differences because they work to reduce $\mathrm{H}_{2} \mathrm{O}_{2}$ to oxygen and water. The decreased CAT expression directly compensates for the decreased ROS activity. While GPx increased levels may mean that, at this wound state, GPx still continues to work for a complete recovery. Therefore, it is important to note that antioxidant enzymes work in a temporal and spatial expression pattern. ${ }^{21)}$ MPO, an antioxidant enzyme which is abundant in neutrophils and a marker of inflammatory infiltration, ${ }^{22)}$ showing decrease levels proves that SAEW is competent in neutrophil infiltration inhibition. Moreover, SAEW mediates the expression of Nrf2, a main regulator of the antioxidant response, ${ }^{23)}$ and AHR, another important signalling for the oxidation and antioxidation which has a power to switch as oxidant through CYP1A1 or an antioxidant through Nrf2 by producing antioxidant enzymes. $^{24)}$ Taken together, antioxidant enzymes seem to regulate the produced ROS, producing oxidative stress balance, leading to wound healing.

NO also plays an important role in wound healing by its influence on inflammation, proliferation, matrix deposition, angiogenesis and remodelling, and on oxidative and nitroxidative stress balance. ${ }^{25,26)}$ Therefore, the higher concentration of NO in SAEW-treated group may indicate its help in facilitating faster wound healing either through phagocytosis or inflammation related mechanism. It can also be possible to be involved in the oxidative stress mechanism as observed in the activities of the antioxidant enzymes. 
The involvement of immune cells in wound healing has been known for the migration of neutrophils, macrophages and lymphocytes into the wound site. ${ }^{10)}$ For instance, macrophages move to the wound through the chemical messengers from the damage cells and platelets wherein they can thrive in the more acidic wound environment. ${ }^{11)}$ Similarly, immune cells in wounded mice might move to the wound area, which might be supported by our previous finding about the correlation between circulating $\mathrm{WBC}$, and its differential counts and the damaged histopathology. This might explain the reduction of WBC count in the circulating serum. However, the absence of histological confirmation is the limitation of this study. To explain the mechanism further, ${ }^{27)}$ the blood's neutrophil count, one of the important markers of inflammation stage, and functions to remove foreign materials such as bacteria, and non-functional host cells from the wound site, ${ }^{27)}$ shows no significant difference among all groups and it might be because the wounds were past the initial inflammatory phase. Other WBC components are lymphocytes, which migrate into the wounds in proliferative stage, peaking on day $7,{ }^{28)}$ and monocytes, which mature into macrophages, and good sources of cytokines and stimulates fibroblast activity, collagen synthesis and angiogenesis. ${ }^{29)}$ Both lymphocytes and monocytes showed a significant decrease compared to the normal groups proving its work in late inflammation to remodelling phase. It is also observed that Betadine and alcohol and the experimental group, SAEW, did not show any significant difference with the negative control, saline, which may be due to innate ability of wounds to heal according to time. In addition, proinflammatory cytokines IL- $1 \beta$, IL- 6 and TNF- $\alpha$, produced by neutrophils, macrophages and keratinocytes, are known to be upregulated during the inflammatory phase ${ }^{30}$ while chemokines, such as $\mathrm{KC}$, is also known to have roles in epithelialization, tissue remodelling, and angiogenesis. ${ }^{31)}$ The observed lower serum IL- $1 \beta$, IL- 6 , TNF- $\alpha$ and $\mathrm{KC}$ concentration may mean that alcohol and SAEW may potentially mediate the activity of these cytokines more in response to wound healing earlier than Betadine and saline treatment. Taken together, there seems to be a quick immune response modulation upon treatment of SAEW causing the wound healing efficacy.

Calcium is identified as a potential central regulator in wound healing in the skin which can be through maintaining normal homeostasis or helps in keratinocyte proliferation and differentiation ${ }^{32)}$ and by producing $\mathrm{H}_{2} \mathrm{O}_{2}$ which promotes healing through the mitogen-activated protein kinase (MAPK) or directly through the transcription genes. ${ }^{33)}$ The reduced amount of calcium concentration in serum can be due to its action to the wound site. Because the wounds were in remodelling phase and towards complete healing, the amount of calcium concentration in skin lysate was back to normal state. Taken together, SAEW-treated group shows to be favourable in mediating intracellular calcium also related to ROS production.

Matrix metalloproteinases and their inhibitors are enzymes which also play a critical role in wound healing by mainly degrading substances in extracellular matrix but is also recently known to be also responsible in inflammation, epithelial repair and resolution, wherein MMP1 and MMP9 were studied to be the major regulators. ${ }^{34)}$ In addition, MMPs activity is controlled by specific locations and stages of wound healing. ${ }^{35)}$ MMP1 facilitates keratinocyte migration and MMP9 promotes cell migration. ${ }^{34,36)}$ Towards complete re-epithelialization, MMP1 and MMP9 should decrease, which was shown in our results upon SAEW treatment, as high levels were evident in chronic wounds. However, this result showed that saline treatment mediated MMP1 expression sooner than the SAEW treatment, while SAEW mediated MMP9 more. Taken together, SAEW seems to be regulating MMP1 and MMP9, at this wound stage, which facilitates faster healing through keratinocyte and cell migration.

Since wound healing is a complex process, it is possible that different factors interact and affect one another to help in faster and effective healing. First, ROS production is influenced by calcium signalling through $\mathrm{H}_{2} \mathrm{O}_{2}$ production leading to immune cell recruitment. ${ }^{28)}$ In addition, calcium and oxidative stress have an effect on extracellular signal-regulated kinase (ERK)-1/2 in the production of MMP-9 for vascular remodelling. ${ }^{37)}$ This cross linking of different factors, plus the earlier mentioned oxidative stress and immune modulation all together can influence wound healing. On the wound healing effect of SAEW, which components would execute the healing mechanism needs to be speculated. Our product is mainly composed of hypochlorous acid (HOCI) wherein we can attribute its effects. Several line of evidences have shown $\mathrm{HOCl}$ as an ideal wound care agent thanks to its powerful microbicidal, anti-biofilm, and wound healing potency. ${ }^{38,39)}$

As a plausible mechanism involving acidic water, acidic water has been known to induce hBD2, irrespective of $\mathrm{NF}-\kappa \mathrm{B} .{ }^{13,14)}$ Strategically, acidic water inhibits NF- $\kappa \mathrm{B}$ activity by attenuating nuclear-cytoplasmic shuttling of p65 and p50 subunits. ${ }^{14)}$ Further, increased NF- $\kappa$ B expression would induce cutaneous inflammation, ${ }^{40)}$ consequently hBD might improve wound healing. ${ }^{41)}$ These potential mechanisms implicated in wound healing remain to be elucidated in our on-going acidic water study. In summary, our results show that SAEW shows favourable results in wound healing via immune-redox modulation and the cross linking of all factors leading to not only its potency in healing wounds, but most especially on its quick action toward wound healing.

\section{CONCLUSION}

Collectively, our study indicates that SAEW is effective in wound healing of hairless mice via immune-redox modulation, and heals better/faster than conventional agents such as alcohol and Betadine. Importantly, this finding might imply the potential use of SAEW as a wound healing agent with more benefit of cost-effectiveness and safety than conventional antiseptics.

Acknowledgment This work was supported by the Ministry of Education of the Republic of Korea and the National Research Foundation of Korea [NRF-2016S1A5B8925203].

Conflict of Interest The authors declare no conflict of interest.

\section{REFERENCES}

1) Young A, McNaught CE. The physiology of wound healing. Surgery, 29, 475-479 (2011).

2) Park JE, Barbul A. Understanding the role of immune regulation in 
wound healing. Am. J. Surg., 187 (5A), S11-S16 (2004).

3) Dunnill C, Patton T, Brennan J, Barrett J, Dryden M, Cooke J, Leaper D, Georgopoulos NT. Reactive oxygen species (ROS) and wound healing: the functional role of ROS and emerging ROSmodulating technologies for augmentation of the healing process. Int. Wound J., 14, 89-96 (2017).

4) Kurahashi T, Fujii J. Roles of antioxidative enzymes in wound healing. J. Dev. Biol., 3, 57-70 (2015).

5) Schreml S, Szeimies RM, Karrer S, Heinlin J, Landthaler M, Babilas $\mathrm{P}$. The impact of the $\mathrm{pH}$ value on skin integrity and cutaneous wound healing. J. Eur. Acad. Dermatol. Venereol., 24, 373-378 (2010).

6) Barrientos S, Stojadinovic O, Golinko MS, Brem H, Tomic-Canic M. Growth factors and cytokines in wound healing. Wound Repair Regen., 16, 585-601 (2008).

7) Nakagawara S, Goto T, Nara M, Ozawa Y, Hotta K, Arata Y. Spectroscopic characterization and the $\mathrm{pH}$ dependence of bactericidal activity of the aqueous chlorine solution. Anal. Sci., 14, 691-698 (1998).

8) Huang YR, Hung YC, Hsu SY, Huang YW, Hwang DF. Application of electrolyzed water in the food industry. Food Contr., 19, 329-345 (2008).

9) Kawada J, Yamada H, Matsuba Y, Ogawa H. Effectiveness of handwashing using acidic electrolyzed water-A comparative study of weak acidic electrolyzed water, strong acidic electrolyzed water and tap water. Skin Res., 42, 137-142 (2000).

10) Lee JH, Rhee PL, Kim JH, Kim JJ, Paik SW, Rhee JC, Song JH, Yeom JS, Lee NY. Efficacy of electrolyzed acid water in reprocessing patient-used flexible upper endoscopes: Comparison with $2 \%$ alkaline glutaraldehyde. J. Gastroenterol. Hepatol., 19, 897-903 (2004).

11) Vorobjeva NV, Vorobjeva LI, Khodjaev EY. The bactericidal effects of electrolyzed oxidizing water on bacterial strains involved in hospital infections. Artif. Organs, 28, 590-592 (2004).

12) Nakae $H$, Inaba $H$. Effectiveness of electrolyzed oxidized water irrigation in a burn-wound infection model. J. Trauma Acute Care Surg., 49, 511-514 (2000).

13) Gojoubori $T$, Nishio $Y$, Asano $M$, Nishida $T$, Komiyama $K$, Ito $\mathrm{K}$. Distinct signaling pathways leading to the induction of human $\beta$-defensin 2 by stimulating an electrolyticaly-generated acid functional water and double strand RNA in oral epithelial cells. J. Recept. Signal Transduct. Res., 34, 97-103 (2014).

14) Gojoubori $\mathrm{T}$, Ota $\mathrm{H}$, Kusunoki M, Nishio $\mathrm{Y}$, Nishio $\mathrm{K}$, Iwasa $\mathrm{S}$, Kaneko Y, Asano M. Electrolytically generated acid functional water inhibits NF- $\kappa \mathrm{B}$ activity by attenuating nuclear-cytoplasmic shuttling of p65 and p50 subunits. J. Recept. Signal Transduct. Res., 36, 248-253 (2016).

15) Xin H, Zheng Y, Hajime N, Han Z. Effect of electrolyzed oxidizing water and hydrocolloid occlusive dressings on excised burn-wounds in rats. Chin. J. Traumatol., 6, 234-237 (2003).

16) Yahagi N, Kono M, Kitahara M, Ohmura A, Sumita O, Hashimoto T, Hori K, Ning-Juan C, Woodson P, Kubota S, Murakami A, Takamoto S. Effect of electrolyzed water on wound healing. Artif. Organs, 24, 984-987 (2000).

17) Walker HL, Mason AD Jr. A standard animal burn. J. Trauma Acute Care Surg., 8, 1049-1051 (1968).

18) Percival SL, McCarty S, Hunt JA, Woods EJ. The effects of pH on wound healing, biofilms, and antimicrobial efficacy. Wound Repair Regen., 22, 174-186 (2014)

19) Goldkorn T, Balaban N, Matsukuma K, Chea V, Gould R, Last J, Chan C, Chavez C. EGF-Receptor phosphorylation and signaling are targeted by $\mathrm{H}_{2} \mathrm{O}_{2}$ redox stress. Am. J. Respir. Cell Mol. Biol., 19,
786-798 (1998).

20) Roy S, Khanna S, Nallu K, Hunt TK, Sen CK. Dermal wound healing is subject to redox control. Mol. Ther., 13, 211-220 (2006).

21) Steiling H, Munz B, Werner S, Brauchle M. Different types of ROS-scavenging enzymes are expressed during cutaneous wound repair. Exp. Cell Res., 247, 484-494 (1999).

22) Hasmann A, Wehrschuetz-Sigl E, Marold A, Wiesbauer H, Schoeftner R, Gewessler U, Kandelbauer A, Schiffer D, Schneider K, Binder B, Schintler M, Guebitz GM. Analysis of myeloperoxidase activity in wound fluids as a marker of infection. Ann. Clin. Biochem., 50, 245-254 (2013).

23) Schäfer M, Werner S. Nrf2 - A regulator of keratinocyte redox signaling. Free Radic. Biol. Med., 88 (Pt B), 243-252 (2015).

24) Furue M, Takahara M, Nakahara T, Uchi H. Role of AhR/ARNT system in skin homeostasis. Arch. Dermatol. Res., 306, 769-779 (2014)

25) Luo J, Chen AF. Nitric oxide: a newly discovered function on wound healing. Acta Pharmacol. Sin., 26, 259-264 (2005).

26) Soneja A, Drews M, Malinski T. Role of nitric oxide, nitroxidative and oxidative stress in wound healing. Pharmacol. Rep., 57 (Suppl.), 108-119 (2005).

27) Diegelmann RF, Evans MC. Wound healing: an overview of acute, fibrotic and delayed healing. Front. Biosci., 9, 283-289 (2004).

28) Fishel RS, Barbul A, Beschorner WE, Wasserkrug HL, Efron G. Lymphocyte participation in wound healing. Morphologic assessment using monoclonal antibodies. Ann. Surg., 206, 25-29 (1987).

29) Enoch S, Leaper DJ. Basic science of wound healing. Surgery, 26, 31-37 (2008).

30) Grellner W, Georg T, Wilske J. Quantitative analysis of proinflammatory cytokines (IL-1 $\beta$, IL-6, TNF- $\alpha$ ) in human skin wounds. Forensic Sci. Int., 113, 251-264 (2000).

31) Ding J, Tredget EE. The role of chemokines in fibrotic wound healing. Adv. Wound Care (New Rochelle), 4, 673-686 (2015).

32) Lansdown AB. Calcium. A potential central regulator in wound healing in the skin. Wound Repair Regen., 10, 271-285 (2002).

33) Cordeiro JV, Jacinto A. The role of transcription-independent damage signals in the initiation of epithelial wound healing. Nat. Rev. Mol. Cell Biol., 14, 249-262 (2013).

34) Caley MP, Martins VL, O'Toole EA. Metalloproteinases and wound healing. Adv. Wound Care, 4, 225-234 (2015).

35) Gill SE, Parks WC. Metalloproteinases and their inhibitors: regulators of wound healing. Int. J. Biochem. Cell Biol., 40, 1334-1347 (2008).

36) Rohani MG, Parks WC. Matrix remodeling by MMPs during wound repair. Matrix Biol., 44-46, 113-121 (2015).

37) Moshal KS, Sen U, Tyagi N, Henderson B, Steed M, Ovechkin AV, Tyagi SC. Regulation of homocysteine-induced MMP-9 by ERK1/2 pathway. Am. J. Physiol. Cell Physiol., 290, C883-C891 (2006).

38) Serhan Sakarya M, Necati Gunay M, Meltem Karakulak M, Barcin Ozturk M, Bulent Ertugrul M. Hypochlorous acid: an ideal wound care agent with powerful microbicidal, antibiofilm, and wound healing potency. Wounds, 26, 342-350 (2014).

39) Armstrong DG, Bohn G, Glat P, Kavros SJ, Kirsner R, Snyder R, Tettelbach W. Expert recommendations for the use of hypochlorous solution: Science and clinical application. Wounds, 61, 2-19 (2015).

40) Wullaert A, Bonnet MC, Pasparakis M. NF- $\kappa$ B in the regulation of epithelial homeostasis and inflammation. Cell Res., 21, 146-158 (2011).

41) Sun H, Wang M, Hao L, Wang J, Su Y, Zou Z. Human beta-defensins improve wound healing through a mechanism affecting multiaspects of healing process. Adv. Wound Care, 2, 149-154 (2011). 